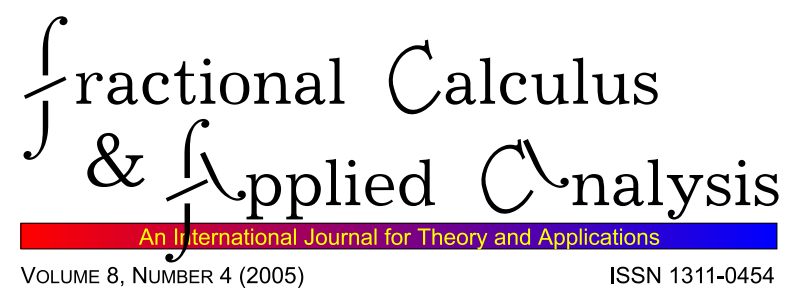

\title{
CAUCHY-TYPE PROBLEM FOR DIFFUSION-WAVE EQUATION WITH THE RIEMANN-LIOUVILLE PARTIAL DERIVATIVE
}

\author{
Anatoly A. Kilbas ${ }^{1}$, Juan J. Trujillo ${ }^{2}$, Aleksandr A. Voroshilov ${ }^{1}$ \\ Dedicated to Professor H.M. Srivastava, \\ on the occasion of his 65th Birth Anniversary
}

\begin{abstract}
The paper is devoted to the study of the Cauchy-type problem for the differential equation

$$
\left(D_{0+, t}^{\alpha} u\right)(x, t)=\lambda^{2} \Delta_{x} u(x, t) \quad\left(x \in \mathbb{R}^{m} ; t>0 ; \lambda>0\right),
$$

involving the Riemann-Liouville partial fractional derivative of order $\alpha>0$

$$
\begin{gathered}
\left(D_{0+, t}^{\alpha} u\right)(x, t)=\left(\frac{\partial}{\partial t}\right)^{n} \frac{1}{\Gamma(n-\alpha)} \int_{0}^{t} \frac{u(x, \tau) d \tau}{(t-\tau)^{\alpha-n+1}} \\
\left(n=[\alpha]+1 ; x \in \mathbb{R}^{m} ; t>0\right),
\end{gathered}
$$

and the Laplace operator $\Delta_{x}=\sum_{j=1}^{m} \frac{\partial^{2} u}{\partial x_{j}^{2}}$ with respect to $x=\left(x_{1}, x_{2}, \ldots, x_{m}\right)$ $\in \mathbb{R}^{m}$. Using the direct and inverse Fourier and Laplace integral transforms, the explicit solution of the considered problem is deduced in terms of the Mittag-Leffler function. In the case $0<\alpha<2$ the explicit solution is established via the $H$-function, in particular via the Wright function for $m=1$, and its asymptotic behavior at infinity is studied. Special cases are considered. Examples are given, and their solutions are illustrated by using the program Mathematica.
\end{abstract}


2000 Mathematics Subject Classification: 35A15, 44A15, 26A33

Key Words and Phrases: diffusion-wave equation of fractional order, Riemann-Liouville partial fractional derivative, Cauchy-type problem, $\mathrm{H}$ function, Mittag-Leffler and Wright functions, Fourier and Laplace transforms

\section{Introduction}

The paper deals with the differential equation

$$
\left(D_{0+, t}^{\alpha} u\right)(x, t)=\lambda^{2} \Delta_{x} u(x, t) \quad\left(x \in \mathbb{R}^{m} ; t>0\right),
$$

where $\lambda>0,\left(D_{0+, t}^{\alpha} u\right)(x, t)$ is the Riemann-Liouville partial fractional derivative of order $\alpha>0$ of a function $u(x, t)$ with respect to the second variable [15, Section 24.1]:

$$
\begin{gathered}
\left(D_{0+, t}^{\alpha} u\right)(x, t)=\left(\frac{\partial}{\partial t}\right)^{n} \frac{1}{\Gamma(n-\alpha)} \int_{0}^{t} \frac{u(x, \tau) d \tau}{(t-\tau)^{\alpha-n+1}} \\
\left(n=[\alpha]+1 ; x \in \mathbb{R}^{m} ; t>0\right),
\end{gathered}
$$

and $\Delta_{x}$ is the Laplace operator with respect to $x=\left(x_{1}, x_{2}, \ldots, x_{m}\right) \in \mathbb{R}^{m}$ : $\Delta_{x}=\sum_{j=1}^{m} \frac{\partial^{2} u}{\partial x_{j}^{2}}$.

When $\alpha=1$ and $\alpha=2$, then

$$
\left(D_{0+, t}^{1} u\right)(x, t)=\frac{\partial u(x, t)}{\partial t} \text { and }\left(D_{0+, t}^{2} u\right)(x, t)=\frac{\partial^{2} u(x, t)}{\partial t^{2}},
$$

respectively, and hence equation (1.1) with $\alpha=1$ and $\alpha=2$ coincide with the diffusion (heat) equation

and the wave equation

$$
\frac{\partial u(x, t)}{\partial t}=\lambda^{2} \Delta_{x} u(x, t) \quad\left(x \in \mathbb{R}^{m} ; t>0\right),
$$

$$
\frac{\partial^{2} u(x, t)}{\partial t^{2}}=\lambda^{2} \Delta_{x} u(x, t) \quad\left(x \in \mathbb{R}^{m} ; t>0\right),
$$

respectively. Therefore equation (1.1) is known as the diffusion-wave equation [12, Section 4.2.2]. In particular, when $m=1$ equation (1.1) takes the form

$$
\left(D_{0+, t}^{\alpha} u\right)(x, t)=\lambda^{2} \frac{\partial^{2} u(x, t)}{\partial x^{2}} \quad(x \in \mathbb{R} ; t>0 ; \lambda>0) .
$$

Equations (1.1) and (1.6) arise in many applications while solving diffusion problems in physics, mechanics and other applied sciences $[12$, Sections 4.2.1 and 4.2.2], [1], [5], [6]; see historical information and a survey of results in the paper $[9$, Ch.7]. 
The present paper is devoted to solution of equation (1.1) of order $\alpha>0$ with the initial conditions

$$
\left(D_{0+, t}^{\alpha-k} u\right)(x, 0+)=f_{k}(x) \quad\left(k=1, \ldots, n=-[-\alpha] ; x \in \mathbb{R}^{m}\right) .
$$

Here $\left(D_{0+, t}^{\alpha-k} u\right)(x, 0+)$ for $n-1<\alpha<n$ is understood in the following sense:

$$
\begin{gathered}
\left(D_{0+, t}^{\alpha-k} u\right)(x, 0+)=\lim _{t \rightarrow 0+}\left(D_{0+, t}^{\alpha-k} u\right)(x, t) \quad(k=1, \ldots, n-1), \\
\left(D_{0+, t}^{\alpha-n} u\right)(x, 0+)=\lim _{t \rightarrow 0+}\left(I_{0+, t}^{n-\alpha} u\right)(x, t)
\end{gathered}
$$

while for $\alpha=n \in \mathbb{N}$

$$
\left(D_{0+, t}^{n-k} u\right)(x, 0+)=\frac{\partial^{n-k}}{\partial t^{n-k}} u(x, 0) \quad(k=1, \ldots, n),
$$

where $\left(I_{0+, t}^{n-\alpha} u\right)(x, t)$ is the Riemann-Liouville partial fractional integral of order $n-\alpha$ [15, Section 24.1]:

$$
\begin{gathered}
\left(I_{0+, t}^{n-\alpha} u\right)(x, t)=\frac{1}{\Gamma(n-\alpha)} \int_{0}^{t} \frac{u(x, \tau) d \tau}{(t-\tau)^{1-n+\alpha} \quad(\alpha<n) ;} \\
\left(I_{0+, t}^{0} u\right)(x, t)=u(x, t) .
\end{gathered}
$$

If $\alpha=n \in \mathbb{N}$, then in accordance with (1.2) and (1.10), problem (1.1), (1.7) takes the form of the Cauchy problem for the differential equation with the partial derivative of order $n$ :

$$
\begin{gathered}
\frac{\partial^{n} u(x, t)}{\partial t^{n}}=\lambda^{2} \Delta_{x} u(x, t) \quad\left(x \in \mathbb{R}^{m}, t>0\right), \\
\frac{\partial^{n-k} u(x, 0)}{\partial t^{n-k}}=f_{k}(x) \quad\left(k=1, \ldots, n ; x \in \mathbb{R}^{m}\right) .
\end{gathered}
$$

Therefore, by an analogy, problem (1.1), (1.7) is called the Cauchy-type problem.

In this paper we apply the direct and inverse Fourier and Laplace integral transforms to establish the solution in closed form of problem (1.1), (1.7). First for any $\alpha>0$ we deduce such a solution in terms of the above transforms and of the Mittag-Leffler function [4, 18.1(19)]:

$$
E_{\alpha, \beta}(z)=\sum_{j=0}^{\infty} \frac{z^{j}}{\Gamma(\alpha j+\beta)} \quad(\alpha>0, \beta>0 ; z \in \mathbb{C}) .
$$


Then we prove that in the case $0<\alpha<2$ the explicit solution can be expressed via the $H_{2,2}^{2,0}$-function. Such a general function $H_{p, q}^{m, n}(z)$ for integers $m, n, p, q(0 \leq m \leq q, 0 \leq n \leq p)$, complex $a_{i}, b_{j} \in \mathbb{C}$ and positive $\alpha_{i}, \beta_{j}(1 \leq i \leq p ; 1 \leq j \leq q)$ is defined by

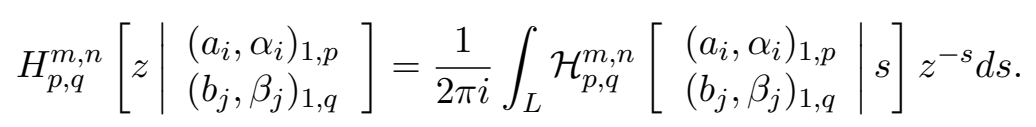

Here

$$
\begin{gathered}
\mathcal{H}(s) \equiv \mathcal{H}_{p, q}^{m, n}\left[\begin{array}{c}
\left(a_{i}, \alpha_{i}\right)_{1, p} \mid \\
\left(b_{j}, \beta_{j}\right)_{1, q}
\end{array} \mid s\right] \\
=\frac{\prod_{j=1}^{m} \Gamma\left(b_{j}+\beta_{j} s\right) \prod_{i=1}^{n} \Gamma\left(1-a_{i}-\alpha_{i} s\right)}{\prod_{i=n+1}^{p} \Gamma\left(a_{i}+\alpha_{i} s\right) \prod_{j=m+1}^{q} \Gamma\left(1-b_{j}-\beta_{j} s\right)}
\end{gathered}
$$

$L$ is specially chosen contour, an empty product, if it occurs, is taken to be one. Note that this function contain most of elementary and special functions; its theory can be found, for example, in the books by Mathai and Saxena [10, Ch.2], Srivastava, Gupta and Goyal [17, Ch.1], Prudnikov, Brychkov and Marichev [14, Sect.8.3] and Kilbas and Saigo [7, Ch.s 1 and 2].

In particular when $m=1$, we show that the obtained solution is given in terms the Wright function $[4,18.1(27)]$ :

$$
\varphi(a, b ; z)=\sum_{j=0}^{\infty} \frac{z^{j}}{j ! \Gamma(a j+b)} \quad(a>0, b>0, z \in \mathbb{C}),
$$

being an entire function of $z$ for $a>-1$ and any $b \in \mathbb{R}$, [8, Corollary 1$]$.

Special cases of the above problems $(1.1),(1.7)$ and $(1.6), 1.7)$ are considered. Using asymptotic estimates for the $H_{p, q}^{m, n}$-function (1.15), we investigate the asymptotic behavior of the obtained explicit solution $u(x . t)$ for $0<\alpha<2$ at infinity.

Finally we consider examples of the Cauchy-type problems (1.1), (1.7) and (1.6), (1.7), and illustrate obtained solutions by using the program Mathematica.

\section{Explicit solutions in terms of the Laplace and Fourier transforms and of the Mittag-Leffler function}

To solve the Cauchy-type problem (1.1), (1.7) we apply the Laplace and Fourier transforms of a function $u(x, t)$ with respect to $t>0$ and $x \in \mathbb{R}^{m}$ : 


$$
\begin{aligned}
& \left(\mathcal{L}_{t} u\right)(x, s)=\int_{0}^{\infty} u(x, t) e^{-s t} d t \quad\left(x \in \mathbb{R}^{m} ; s \in \mathbb{C}\right), \\
& \left(\mathcal{F}_{x} u\right)(\sigma, t)=\int_{\mathbb{R}^{m}} u(x, t) e^{i x \cdot \sigma} d x \quad\left(\sigma \in \mathbb{R}^{m} ; t>0\right),
\end{aligned}
$$

and their inverse transforms with respect to $s \in \mathbb{C}$ and $\sigma \in \mathbb{R}^{m}$ :

$$
\begin{array}{r}
\left(\mathcal{L}_{s}^{-1} u\right)(x, t)=\frac{1}{2 \pi i} \int_{\gamma-i \infty}^{\gamma+i \infty} e^{s t} u(x, s) d s \quad\left(x \in \mathbb{R}^{m} ; t>0\right), \\
\left(\mathcal{F}_{\sigma}^{-1} u\right)(x, t)=\frac{1}{(2 \pi)^{m}} \int_{\mathbb{R}^{m}} u(\sigma, t) e^{-i \sigma \cdot x} d \sigma \quad\left(x \in \mathbb{R}^{m} ; t>0\right) .
\end{array}
$$

Here $x \cdot \sigma=\sum_{i=1}^{m} x_{i} \sigma_{i}$ for $x=\left(x_{1}, \ldots, x_{m}\right) \in \mathbb{R}^{m}$ and $\sigma=\left(\sigma_{1}, \ldots, \sigma_{m}\right) \in \mathbb{R}^{m}$, while $\gamma \in \mathbb{R}$ is a fixed real number. One may find properties of the direct and inverse Laplace and Fourier transforms, for example, in books by Ditkin and Prudnikov [2, Ch.II], Sneddon [16, Ch.1, §4] and by Stein and Weiss [18] and Nikol'skii [11, Ch.16]. In particular, each of the transforms (2.1), (2.3) and (2.2), (2.4) is inverse to the other one for suitable functions $u(x, t)$.

Suppose that there exist the Fourier transforms $\left(\mathcal{F}_{x} f_{k}\right)(\sigma)$ of $f_{k}(x)$ $(k=1, \ldots, n)$ in (1.7). Applying the Laplace transform (2.1) to both sides of equation (1.1), taking the initial conditions (1.7) and the formula for the Laplace transform of the Riemann-Liouville partial fractional derivative [12, (2.248)]:

$$
\left(\mathcal{L}_{t} D_{0+, t}^{\alpha} u\right)(x, s)=s^{\alpha}\left(\mathcal{L}_{t} u\right)(x, s)-\sum_{k=1}^{n} s^{k-1}\left(D_{0+, t}^{\alpha-k} u\right)(x, 0+),
$$

we have

$$
s^{\alpha}\left(\mathcal{L}_{t} u\right)(x, s)-\sum_{k=1}^{n} s^{k-1} f_{k}(x)=\lambda^{2}\left(\Delta_{x} \mathcal{L}_{t} u\right)(x, s) .
$$

Applying the Fourier transform (2.2) to (2.6) and using the formula for the Fourier transform of the operator $\Delta_{x}$ :

$$
\left(\mathcal{F}_{x} \Delta_{x} u\right)(\sigma, s)=-|\sigma|^{2}\left(\mathcal{F}_{x} u\right)(\sigma, s),
$$

we obtain the following relation

$$
\left(\mathcal{F}_{x} \mathcal{L}_{t} u\right)(\sigma, s)=\sum_{k=1}^{n} \frac{s^{k-1}}{s^{\alpha}+\lambda^{2}|\sigma|^{2}}\left(\mathcal{F}_{x} f_{k}\right)(\sigma) \quad\left(\sigma \in \mathbb{R}^{m} ; s \in \mathbb{C}\right),
$$


where $|\sigma|^{2}=\sum_{i=1}^{m} \sigma_{i}^{2}$.

Applying the inverse Fourier and Laplace transforms (2.4) and (2.3), we deduce solution $u(x, t)$ of the initial problem $(1.1),(1.7)$ in the form:

$$
u(x, t)=\left(\mathcal{L}_{s}^{-1} \mathcal{F}_{\sigma}^{-1}\left[\sum_{k=1}^{n} \frac{s^{k-1}}{s^{\alpha}+\lambda^{2}|\sigma|^{2}}\left(\mathcal{F}_{x} f_{k}\right)(\sigma)\right]\right)(x, t) .
$$

We express solution (2.9) in terms of the Mittag-Leffler function (1.14), being an entire function of $z \in \mathbb{C}$. On the basis of the formula for the Laplace transform of this function(for example, see [15, (1.93)], there hold the relations

$$
\begin{gathered}
\left(\mathcal{L}_{t}\left[t^{\alpha-k} E_{\alpha, \alpha-k+1}\left(-\lambda^{2}|\sigma|^{2} t^{\alpha}\right)\right]\right)(s)=\frac{s^{k-1}}{s^{\alpha}+\lambda^{2}|\sigma|^{2}} \\
\left(k=1, \ldots, n ; s \in \mathbb{C}, \sigma \in \mathbb{R}^{m}, \lambda>0 ; \lambda|\sigma||s|^{-2 / \alpha}<1\right) .
\end{gathered}
$$

Applying the inverse Laplace and Fourier transforms (2.3) and (2.4) to (2.8) and taking (2.10) into account, we obtain the explicit solution of the problem (1.1), (1.7) in the form

$$
u(x, t)=\sum_{k=1}^{n} \frac{t^{\alpha-k}}{(2 \pi)^{m}} \int_{\mathbb{R}^{m}} E_{\alpha, \alpha-k+1}\left(-\lambda^{2}|\sigma|^{2} t^{\alpha}\right)\left(\mathcal{F}_{x} f_{k}\right)(\sigma) e^{-i x \cdot \sigma} d \sigma .
$$

The above yields the following result.

Theorem 1. Let $\alpha>0, n=-[-\alpha], m \in \mathbb{N}$ and $\lambda>0$. Let there exist the Fourier transforms $\left(\mathcal{F}_{x} f_{k}\right)(\sigma)$ of functions $f_{k}(x)(k=1, \ldots, n)$, and let the integrals in the right-hand side of (2.11) be convergent. Then the Cauchy-type problem (1.1), (1.7) is solvable, and its explicit solution is given by (2.11).

In particular, when $m=1$, the solution of problem (1.6), (1.7) has the form

$$
u(x, t)=\sum_{k=1}^{n} \frac{t^{\alpha-k}}{2 \pi} \int_{\mathbb{R}} E_{\alpha, \alpha-k+1}\left(-\lambda^{2}|\sigma|^{2} t^{\alpha}\right)\left(\mathcal{F}_{x} f_{k}\right)(\sigma) e^{-i x \sigma} d \sigma .
$$

Corollary 1. Let $n, m \in \mathbb{N}$ and $\lambda>0$. Solution of the Cauchy problem (1.12), (1.13) is given by relation (2.11) with $\alpha=n$ provided that the integrals in the right-hand side of (2.11) are convergent. 
Corollary 2. Let $0<\alpha<1, m \in \mathbb{N}$ and $\lambda>0$, and let there exist the Fourier transforms $\left(\mathcal{F}_{x} f\right)(\sigma)$ of a function $f(x)$. Then solution of the Cauchy-type problem

$$
\left(D_{0+, t}^{\alpha} u\right)(x, t)=\lambda^{2} \Delta_{x} u(x, t), \quad\left(I_{0+, t}^{1-\alpha} u\right)(x, 0+)=f(x) \quad\left(x \in \mathbb{R}^{m} ; t>0\right)
$$

is given by

$$
u(x, t)=\frac{t^{\alpha-1}}{(2 \pi)^{m}} \int_{\mathbb{R}^{m}} E_{\alpha, \alpha}\left(-\lambda^{2}|\sigma|^{2} t^{\alpha}\right)\left(\mathcal{F}_{x} f\right)(\sigma) e^{-i x \cdot \sigma} d \sigma
$$

provided that the integral in the right-hand side of (2.14) is convergent.

In particular, for $m=1$ the solution of the problem

$$
\left(D_{0+, t}^{\alpha} u\right)(x, t)=\lambda^{2} \frac{\partial^{2} u(x, t)}{\partial x^{2}}, \quad\left(D_{0+, t}^{\alpha-1} u\right)(x, 0+)=f(x) \quad(x \in \mathbb{R} ; t>0)
$$

has the form

$$
u(x, t)=\frac{t^{\alpha-1}}{2 \pi} \int_{\mathbb{R}} E_{\alpha, \alpha}\left(-\lambda^{2}|\sigma|^{2} t^{\alpha}\right)\left(\mathcal{F}_{x} f\right)(\sigma) e^{-i x \sigma} d \sigma .
$$

Corollary 3. Let $1<\alpha<2, m \in \mathbb{N}, \lambda>0$, and let there exist the Fourier transforms $\left(\mathcal{F}_{x} f\right)(\sigma)$ and $\left(\mathcal{F}_{x} g\right)(\sigma)$ of functions $f(x)$ and $g(x)$. Then the solution of the Cauchy-type problem for equation (1.1) with the initial conditions

$$
\left(D_{0+, t}^{\alpha-1} u\right)(x, 0+)=f(x), \quad\left(D_{0+, t}^{\alpha-2} u\right)(x, 0+)=g(x) \quad\left(x \in \mathbb{R}^{m} ; t>0\right)
$$

is given by

$$
\begin{gathered}
u(x, t)=\frac{t^{\alpha-1}}{(2 \pi)^{m}} \int_{\mathbb{R}^{m}} E_{\alpha, \alpha}\left(-\lambda^{2}|\sigma|^{2} t^{\alpha}\right)\left(\mathcal{F}_{x} f\right)(\sigma) e^{-i x \cdot \sigma} d \sigma \\
+\frac{t^{\alpha-2}}{(2 \pi)^{m}} \int_{\mathbb{R}^{m}} E_{\alpha, \alpha-1}\left(-\lambda^{2}|\sigma|^{2} t^{\alpha}\right)\left(\mathcal{F}_{x} g\right)(\sigma) e^{-i x \cdot \sigma} d \sigma,
\end{gathered}
$$

provided that integrals in the right-hand side of (2.18) are convergent.

In particular, for $m=1$ the solution of problem (1.6), (2.17) has the form

$$
u(x, t)=\frac{t^{\alpha-1}}{2 \pi} \int_{\mathbb{R}} E_{\alpha, \alpha}\left(-\lambda^{2}|\sigma|^{2} t^{\alpha}\right)\left(\mathcal{F}_{x} f\right)(\sigma) e^{-i x \sigma} d \sigma
$$




$$
+\frac{t^{\alpha-2}}{2 \pi} \int_{\mathbb{R}} E_{\alpha, \alpha-1}\left(-\lambda^{2}|\sigma|^{2} t^{\alpha}\right)\left(\mathcal{F}_{x} g\right)(\sigma) e^{-i x \sigma} d \sigma
$$

Suppose that the functions $f_{k}(x)(k=1, \ldots, n)$ are infinitely differentiable on $\mathbb{R}^{m}$. Substituting (1.14) into (2.11), and changing the orders of integration and summation (it is possible because the series in (1.14) is uniformly convergent), and taking into account the relation

$$
\left(\Delta_{x}^{j} f_{k}\right)(x)=\frac{1}{(2 \pi)^{m}} \int_{\mathbb{R}^{m}}\left(-|\sigma|^{2}\right)^{j}\left(\mathcal{F}_{x} f_{k}\right)(\sigma) e^{-i \sigma \cdot x} d \sigma \quad(j=1,2, \ldots)
$$

for $j$ th powers of the Laplace operator $\left(\Delta_{x}^{1}=\Delta_{x}, \Delta_{x}^{j}=\Delta_{x}^{1} \Delta_{x}^{j-1}, j=\right.$ $2,3, \ldots)$, we obtain the following representation for the solution (2.11):

$$
u(x, t)=\sum_{k=1}^{n} t^{\alpha-k} \sum_{j=0}^{\infty} \frac{\left(\lambda^{2} t^{\alpha}\right)^{j}}{\Gamma(\alpha j+\alpha-k+1)}\left(\Delta_{x}^{j} f_{k}\right)(x) .
$$

This lead us to the next statement.

Theorem 2. Let $\alpha>0, n=-[-\alpha], m \in \mathbb{N}$ and $\lambda>0$. Let functions $f_{k}(x)(k=1, \ldots, n)$ be infinitely differentiable on $\mathbb{R}^{m}$, and let series in the right-hand side of (2.21) be convergent. Then the Cauchy-type problem (1.1), (1.7) is solvable, and its explicit solution is given by (2.21).

In particular, when $m=1$, the solution of problem (1.6), (1.7) has the form

$$
u(x, t)=\sum_{k=1}^{n} t^{\alpha-k} \sum_{j=0}^{\infty} \frac{\left(\lambda^{2} t^{\alpha}\right)^{j}}{\Gamma(\alpha j+\alpha-k+1)} f_{k}^{(2 j)}(x) .
$$

Corollary 4. Let $n, m \in \mathbb{N}$ and $\lambda>0$. Solution of the Cauchy problem (1.12), (1.13) is given by relation (2.21) with $\alpha=n$ provided that the series in the right-hand side of (2.21) are convergent.

Corollary 5. Let $0<\alpha<1, m \in \mathbb{N}$ and $\lambda>0$, and let $f(x)$ be infinitely differentiable function on $\mathbb{R}^{m}$. Then the solution of the Cauchytype problem (2.13) is given by

$$
u(x, t)=t^{\alpha-1} \sum_{j=0}^{\infty} \frac{\left(\lambda^{2} t^{\alpha}\right)^{j}}{\Gamma(\alpha j+\alpha)}\left(\Delta_{x}^{j} f_{k}\right)(x),
$$

provided that the series in the right-hand side of (2.23) is convergent.

In particular, for $m=1$ the solution of the problem (2.15) has the form 


$$
u(x, t)=t^{\alpha-1} \sum_{j=0}^{\infty} \frac{\left(\lambda^{2} t^{\alpha}\right)^{j}}{\Gamma(\alpha j+\alpha-k+1)} f_{k}^{(2 j)}(x) .
$$

Corollary 6. Let $1<\alpha<2, m \in \mathbb{N}, \lambda>0$, and let $f(x)$ and $g(x)$ be infinitely differentiable functions on $\mathbb{R}^{m}$. Then the solution of the Cauchy-type problem (1.1), (2.17) is given by

$$
u(x, t)=t^{\alpha-1} \sum_{j=0}^{\infty} \frac{\left(\lambda^{2} t^{\alpha}\right)^{j}}{\Gamma(\alpha j+\alpha)}\left(\Delta_{x}^{j} f\right)(x)+t^{\alpha-2} \sum_{j=0}^{\infty} \frac{\left(\lambda^{2} t^{\alpha}\right)^{j}}{\Gamma(\alpha j+\alpha-1)}\left(\Delta_{x}^{j} g\right)(x),
$$

provided that the series in the right-hand side of (2.25) are convergent.

In particular, for $m=1$ the solution of problem (1.6), (2.17) has the form

$$
u(x, t)=t^{\alpha-1} \sum_{j=0}^{\infty} \frac{\left(\lambda^{2} t^{\alpha}\right)^{j}}{\Gamma(\alpha j+\alpha)} f^{(2 j)}(x)+t^{\alpha-2} \sum_{j=0}^{\infty} \frac{\left(\lambda^{2} t^{\alpha}\right)^{j}}{\Gamma(\alpha j+\alpha-1)} g^{(2 j)}(x) .
$$

\section{Explicit solutions in terms of the $H$-function}

If $0<\alpha<2$, then the explicit solution of equation (1.1) with initial conditions (1.7) for $0<\alpha \leq 1, n=1$ and $1<\alpha<2, n=2$ are expressed via the $H_{2,2}^{2,0}$-function (1.15). The proof is based on application of the inverse Fourier and Laplace transforms (2.4) and (2.3) to (2.8) and formulas of the Fourier and Laplace transforms of the modified Bessel function of the third kind (McDonald function) $K_{\nu}(z)[3,7.2(13)]$ and of the $H$-function (1.15) of the form

$$
H_{2,2}^{2,0}\left[z \mid \begin{array}{c}
\left(a, \frac{1}{2}\right),\left(b, \frac{\alpha}{2}\right) \\
(c, 1)\left(d, \frac{1}{2}\right)
\end{array}\right]=\frac{1}{2 \pi i} \int_{L} \frac{\Gamma(c+\tau) \Gamma(d+\tau / 2)}{\Gamma(a+\tau / 2) \Gamma(b+\alpha \tau / 2)} z^{-\tau} d \tau .
$$

Here $a, b, c, d \in \mathbb{R}$, and $L$ is a contour starting at the point $\gamma-i \infty$ and terminating at the point $\gamma+i \infty$, where $\gamma \in \mathbb{R}$, and separating all poles of the Gamma-functions $\Gamma(c+\tau)$ and $\Gamma(d+\tau / 2)$ from to the left.

Lemma 1. Let $0<\alpha<2$, let $a, b, c, d, \in \mathbb{R}$, and let $z \in \mathbb{C},|\arg (z)|<$ $(2-\alpha) \pi / 4, z \neq 0$. Then the $H_{2,2}^{2,0}$-function in (3.1) exists. 
$\mathrm{P} \mathrm{r}$ o o f. The result follows from the existence theorem for the $H$ function; see [7, Theorem 1.1].

Lemma 2. For $m \in \mathbb{N}$ and $c>0$ there holds the following formula

$$
\left(\mathcal{F}_{x}\left[|x|^{(2-m) / 2} K_{(m-2) / 2}(c|x|)\right]\right)(\sigma)=\left(\frac{2 \pi}{c}\right)^{m / 2} \frac{c}{c^{2}+|\sigma|^{2}} \quad\left(\sigma \in \mathbb{R}^{m}\right) .
$$

$\mathrm{P}$ r o o f. It is known the following formula of the Fourier transform (2.2) of a radial function on $\mathbb{R}^{m}$ (for example, see [15, (25.11)]:

$$
\left(\mathcal{F}_{x}[\varphi(|x|)]\right)(\sigma)=\frac{(2 \pi)^{\frac{m}{2}}}{|\sigma|^{\frac{m-2}{2}}} \int_{0}^{\infty} \varphi(\rho) \rho^{\frac{m}{2}} J_{\frac{m-2}{2}}(\rho|\sigma|) d \rho .
$$

Setting in $(3.3) \varphi(\rho)=\rho^{\frac{2-m}{2}} K_{\frac{m-2}{2}}(c \rho)$, we have

$$
\begin{gathered}
\left(\mathcal{F}_{x}\left[|x|^{(2-m) / 2} K_{(m-2) / 2}(c|x|)\right]\right)(\sigma) \\
=\frac{(2 \pi)^{\frac{m}{2}}}{|\sigma|^{\frac{m-2}{2}}} \int_{0}^{\infty} \rho^{\frac{2-m}{2}} K_{\frac{m-2}{2}}(c \rho) \rho^{\frac{m}{2}} J_{\frac{m-2}{2}}(\rho|\sigma|) d \rho \\
=\frac{(2 \pi)^{\frac{m}{2}}}{|\sigma|^{\frac{m-2}{2}}} \int_{0}^{\infty} \rho K_{\frac{m-2}{2}}(c \rho) J_{\frac{m-2}{2}}(|\sigma| \rho) d \rho .
\end{gathered}
$$

Using the formula $[13,2.16 .21 .1]$ :

$$
\int_{0}^{\infty} \rho K_{\nu}(a \rho) J_{\nu}(b \rho) d \rho=\left(\frac{b}{a}\right)^{\nu} \frac{1}{a^{2}+b^{2}} \quad(a>0, b \geq 0, \nu>-1)
$$

and setting $a=c>0, b=|\sigma| \geq 0, \nu=\frac{m-2}{2}>-1$, we find

$$
\int_{0}^{\infty} \rho K_{\frac{m-2}{2}}(c \rho) J_{\frac{m-2}{2}}(\rho|\sigma|) d \rho=\left(\frac{|\sigma|}{c}\right)^{\frac{m-2}{2}} \frac{1}{c^{2}+|\sigma|^{2}} .
$$

Substituting (3.6) into (3.4), we obtain (3.2), and thus the lemma is proved. 
According to (3.2) with $c=s^{\alpha / 2} / \lambda$,

$$
\begin{gathered}
\left(\mathcal{F}_{x}\left[|x|^{(2-m) / 2} K_{(m-2) / 2}\left(\frac{s^{\frac{\alpha}{2}}}{\lambda}|x|\right)\right]\right)(\sigma) \\
=(2 \pi)^{\frac{m}{2}} s^{\frac{\alpha(2-m)}{4}} \lambda^{\frac{m+2}{2}} \frac{1}{s^{\alpha}+\lambda^{2}|\sigma|^{2}}
\end{gathered}
$$

and hence

$$
\frac{s^{k-1}}{s^{\alpha}+\lambda^{2}|\sigma|^{2}}=\left(\mathcal{F}_{x}\left[\sum_{k=1}^{n} \frac{s^{\frac{\alpha(m-2)}{4}+k-1}}{\lambda(2 \pi \lambda)^{\frac{m}{2}}}|x|^{\frac{2-m}{2}} K_{\frac{m-2}{2}}\left(\frac{|x|}{\lambda} s^{\frac{\alpha}{2}}\right)\right]\right)(\sigma) .
$$

By (3.7), equality (2.8) takes the form

$$
\begin{gathered}
\left(\mathcal{F}_{x} \mathcal{L}_{t} u\right)(\sigma, s) \\
=\left(\mathcal{F}_{x}\left[\sum_{k=1}^{n} \frac{s^{\frac{\alpha(m-2)}{4}+k-1}}{\lambda(2 \pi \lambda)^{\frac{m}{2}}}|x|^{\frac{2-m}{2}} K_{\frac{m-2}{2}}\left(\frac{|x|}{\lambda} s^{\frac{\alpha}{2}}\right)\right]\right)(\sigma)\left(\mathcal{F}_{x} f_{k}\right)(\sigma) .
\end{gathered}
$$

In accordance with the Fourier convolution theorem, we rewrite this relation in the form

$$
\begin{gathered}
\left(\mathcal{F}_{x} \mathcal{L}_{t} u\right)(\sigma, s) \\
=\left(\mathcal{F}_{x}\left[\sum_{k=1}^{n} \frac{s^{\frac{\alpha(m-2)}{4}+k-1}}{\lambda(2 \pi \lambda)^{\frac{m}{2}}}|x|^{\frac{2-m}{2}} K_{\frac{m-2}{2}}\left(\frac{|x|}{\lambda} s^{\frac{\alpha}{2}}\right) *_{x} f_{k}(x)\right]\right)(\sigma) .
\end{gathered}
$$

Applying the inverse Fourier transform (2.4) to (3.8), we have for $x \in \mathbb{R}^{m}$ and $s \in \mathbb{C}$ :

$$
\left(\mathcal{L}_{t} u\right)(x, s)=\sum_{k=1}^{n} \frac{s^{\frac{\alpha(m-2)}{4}+k-1}}{\lambda(2 \pi \lambda)^{\frac{m}{2}}}|x|^{\frac{2-m}{2}} K_{\frac{m-2}{2}}\left(\frac{|x|}{\lambda} s^{\frac{\alpha}{2}}\right) *_{x} f_{k}(x) .
$$

Applying the inverse Laplace transform (2.3) to (3.9), we can deduce the explicit solution of the Cauchy-type problem (1.1), (1.7). For this we need to find the inverse Laplace transform of the functions

$$
s^{\frac{\alpha(m-2)}{4}+k-1} K_{\frac{m-2}{2}}\left(\frac{|x|}{\lambda} s^{\frac{\alpha}{2}}\right) \quad(k=1, \ldots, n) .
$$

Now we show that for $0<\alpha \leq 1, k=1$ and $1<\alpha<2, k=1,2$ functions in (3.10) are represented by the Laplace transform (2.1) of $H_{2,2}^{2.0}$-functions (3.1) multiplying by power functions. 
Lemma 3. If $0<\alpha \leq 1, k=1$ or $1<\alpha<2, k=1,2$, then

$$
\begin{gathered}
\left(\mathcal{L}_{t}\left[t^{-k-\frac{\alpha(m-2)}{4}} H_{2,2}^{2,0}\left[\frac{|x|}{\lambda} t^{-\frac{\alpha}{2}} \mid \begin{array}{c}
\left(\frac{m}{4}, \frac{1}{2}\right),\left(1-k-\frac{\alpha(m-2)}{4}, \frac{\alpha}{2}\right) \\
\left(\frac{m}{2}-1,1\right),\left(\frac{1}{2}-\frac{m}{4}, \frac{1}{2}\right)
\end{array}\right]\right]\right)(s) \\
=2^{\frac{m}{2}} \pi^{-\frac{1}{2}} s^{\frac{\alpha(m-2)}{4}+k-1} K_{\frac{m-2}{2}}\left(\frac{|x|}{\lambda} s^{\frac{\alpha}{2}}\right) .
\end{gathered}
$$

P r o o f. We use representation (3.1) and chose the contour $L$ in such way that $\operatorname{Re}(\tau)>\frac{m-2}{2}+\frac{2}{\alpha}$. Then $-k-\frac{\alpha(m-2)}{4}+\frac{\alpha}{2} \operatorname{Re}(\tau)>-1$ for $0<\alpha \leq 1$, $k=1$ and $1<\alpha<2, k=1,2$, and this ensure the convergence of all improper integrals below.

From (2.1) and (3.1) we have

$$
\begin{gathered}
\left(\mathcal{L}_{t}\left[t^{-k-\frac{\alpha(m-2)}{4}} H_{2,2}^{2,0}\left[\frac{|x|}{\lambda} t^{-\frac{\alpha}{2}} \mid \begin{array}{c}
\left(\frac{m}{4}, \frac{1}{2}\right),\left(1-k-\frac{\alpha(m-2)}{4}, \frac{\alpha}{2}\right) \\
\left(\frac{m}{2}-1,1\right),\left(\frac{1}{2}-\frac{m}{4}, \frac{1}{2}\right)
\end{array}\right]\right]\right)(s \\
=\frac{1}{2 \pi i} \int_{L} \frac{\Gamma\left(\frac{m}{2}-1+\tau\right) \Gamma\left(\frac{1}{2}-\frac{m}{4}+\frac{\tau}{2}\right)}{\Gamma\left(\frac{m}{4}+\frac{\tau}{2}\right) \Gamma\left(1-k-\frac{\alpha(m-2)}{4}+\frac{\alpha}{2} \tau\right)}\left(\frac{|x|}{\lambda}\right)^{-\tau} d \tau \\
\times \int_{0}^{\infty} e^{-s t} t^{-k-\frac{\alpha(m-2)}{4}+\frac{\alpha}{2} \tau} d t \\
=s^{k-1+\frac{\alpha(m-2)}{4} \frac{1}{2 \pi i}} \int_{L} \frac{\Gamma\left(\frac{m}{2}-1+\tau\right) \Gamma\left(\frac{1}{2}-\frac{m}{4}+\frac{\tau}{2}\right)}{\Gamma\left(\frac{m}{4}+\frac{\tau}{2}\right)}\left(\frac{|x|}{\lambda} s^{\frac{\alpha}{2}}\right)^{-\tau} d \tau
\end{gathered}
$$

Hence, in accordance with (1.15),

$$
\begin{gathered}
\left(\mathcal { L } _ { t } \left[t ^ { - k - \frac { \alpha ( m - 2 ) } { 4 } } H _ { 2 , 2 } ^ { 2 , 0 } \left[\frac{|x|}{\lambda} t^{-\frac{\alpha}{2}}\right.\right.\right. \\
\left.\left.\left.\begin{array}{c}
\left(\frac{m}{4}, \frac{1}{2}\right),\left(1-k-\frac{\alpha(m-2)}{4}, \frac{\alpha}{2}\right) \\
\left(\frac{m}{2}-1,1\right),\left(\frac{1}{2}-\frac{m}{4}, \frac{1}{2}\right)
\end{array}\right]\right]\right) \\
=s^{k-1+\frac{\alpha(m-2)}{4}} H_{1,2}^{2,0}\left[\frac{|x|}{\lambda} s^{\frac{\alpha}{2}}\right. \\
\left(\frac{m}{2}-1,1\right),\left(\frac{m}{4}, \frac{1}{2}\right)
\end{gathered}
$$

It is directly verified the following relation

$$
H_{1,2}^{2,0}\left[\frac{|x|}{\lambda} s^{\frac{\alpha}{2}} \mid \begin{array}{c}
\left(\frac{m}{4}, \frac{1}{2}\right) \\
\left(\frac{m}{2}-1,1\right),\left(\frac{1}{2}-\frac{m}{4}, \frac{1}{2}\right)
\end{array}\right]=2^{\frac{m}{2}} \pi^{-\frac{1}{2}} K_{\frac{m-2}{2}}\left(\frac{|x|}{\lambda} s^{\frac{\alpha}{2}}\right) .
$$


Substitution of (3.13) into (3.12) yields (3.11), which completes the proof of lemma.

Let $n=1$ for $0<\alpha \leq 1$ and $n=2$ for $1<\alpha<2$. Using (3.11), we rewrite (3.9) in the form

$$
\begin{aligned}
& \left(\mathcal{L}_{t} u\right)(x, s)=\frac{2^{-m}|x|^{\frac{2-m}{2}}}{\lambda^{1+\frac{m}{2}} \pi^{\frac{m-1}{2}}}\left(\mathcal { L } _ { t } \left[\sum_{k=1}^{n} t^{-k-\frac{\alpha(m-2)}{4}}\right.\right.
\end{aligned}
$$

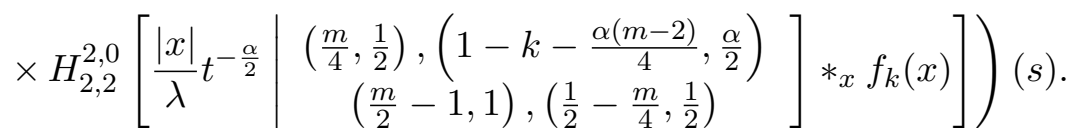

Applying the inverse Laplace transform (2.3), we obtain the solution $u(x, t)$ of the problem (1.1), (1.7) in closed form:

$$
\begin{aligned}
& u(x, t)=\frac{2^{-m}|x|^{\frac{2-m}{2}}}{\lambda^{1+\frac{m}{2}} \pi^{\frac{m-1}{2}}} \\
& \times \sum_{k=1}^{n} t^{-k-\frac{\alpha(m-2)}{4}} H_{2,2}^{2,0}\left[\frac{|x|}{\lambda} t^{-\frac{\alpha}{2}} \mid \begin{array}{c}
\left(\frac{m}{4}, \frac{1}{2}\right),\left(1-k-\frac{\alpha(m-2)}{4}, \frac{\alpha}{2}\right) \\
\left(\frac{m}{2}-1,1\right),\left(\frac{1}{2}-\frac{m}{4}, \frac{1}{2}\right)
\end{array}\right] *_{x} f_{k}(x) .
\end{aligned}
$$

From the above arguments we deduce the following results.

Theorem 3. Let $0<\alpha \leq 1, m \in \mathbb{N}$ and $\lambda>0$. Then the Cauchy-type problem

$$
\left(D_{0+, t}^{\alpha} u\right)(x, t)=\lambda^{2}\left(\Delta_{x} u\right)(x, t), \quad\left(D_{0+, t}^{\alpha-1} u\right)(x, 0+)=f(x) \quad\left(x \in \mathbb{R}^{m}\right)
$$

is solvable, and its explicit solution has the form

$$
u(x, t)=\int_{\mathbb{R}^{m}} G_{1}^{\alpha}(x-\tau, t) f(\tau) d \tau,
$$

where

$$
\begin{aligned}
& G_{1}^{\alpha}(x, t)=\frac{2^{-m}|x|^{\frac{2-m}{2}}}{\lambda^{1+\frac{m}{2}} \pi^{\frac{m-1}{2}}} t^{-1-\frac{\alpha(m-2)}{4}} \\
& \times H_{2,2}^{2,0}\left[\begin{array}{l|l}
\frac{|x|}{\lambda} t^{-\frac{\alpha}{2}} & \begin{array}{c}
\left(\frac{m}{4}, \frac{1}{2}\right),\left(-\frac{\alpha(m-2)}{4}, \frac{\alpha}{2}\right) \\
\left(\frac{m}{2}-1,1\right),\left(\frac{1}{2}-\frac{m}{4}, \frac{1}{2}\right)
\end{array}
\end{array}\right],
\end{aligned}
$$

provided that the integral in the right-hand side of (3.16) is convergent. 
Theorem 4. Let $1<\alpha<2, m \in \mathbb{N}$ and $\lambda>0$. Then the Cauchy-type problem for equation (1.1) with the initial conditions

$$
\left(D_{0+, t}^{\alpha-1} u\right)(x, 0+)=f(x), \quad\left(D_{0+, t}^{\alpha-2} u\right)(x, 0+)=g(x) \quad\left(x \in \mathbb{R}^{m}\right)
$$

is solvable and its explicit solution has the form

$$
u(x, t)=\int_{\mathbb{R}^{m}}\left[G_{1}^{\alpha}(x-\tau, t) f(\tau)+G_{2}^{\alpha}(x-\tau, t) g(\tau)\right] d \tau,
$$

where $G_{1}^{\alpha}(x, t)$ is given by (3.17), and

$$
\begin{gathered}
G_{2}^{\alpha}(x, t)=\frac{2^{-m}|x|^{\frac{2-m}{2}}}{\lambda^{1+\frac{m}{2}} \pi^{\frac{m-1}{2}}} t^{-2-\frac{\alpha(m-2)}{4}} \\
\times H_{2,2}^{2,0}\left[\begin{array}{l|c}
\frac{|x|}{\lambda} t^{-\frac{\alpha}{2}} & \left.\begin{array}{c}
\left(\frac{m}{4}, \frac{1}{2}\right),\left(-1-\frac{\alpha(m-2)}{4}, \frac{\alpha}{2}\right) \\
\left(\frac{m}{2}-1,1\right),\left(\frac{1}{2}-\frac{m}{4}, \frac{1}{2}\right)
\end{array}\right],
\end{array}\right.
\end{gathered}
$$

provided that the integral in the right-hand side of (3.19) is convergent.

REMARK 1. Hilfer [6] considered problem (3.15) when $f(x)=\delta(x)$ is the Dirac delta function, and expressed its the so-called fundamental solution in terms of the $H_{1,2}^{2,0}$-function.

REMARK 2. Functions $G_{1}^{\alpha}(x, t)$ and $G_{2}^{\alpha}(x, t)$ in (3.17) and (3.20) are called the fractional Green functions by an analogy with the ordinary case: see [12, Section 4.1].

\section{Explicit solutions in terms of the Wright function}

When $m=1, H_{2,2}^{2,0}$-functions in (3.14) are expressed via the Wright function (1.17).

Lemma 4. Let $0<\alpha<2, \lambda>0$ and let $k=1$ for $0<\alpha \leq 1$, while $k=1,2$ for $1<\alpha<2$. Then for $x \in \mathbb{R}$ and $t>0$

$$
\begin{gathered}
H_{2,2}^{2,0}\left[\frac{|x|}{\lambda} t^{-\frac{\alpha}{2}} \mid \begin{array}{c}
\left(\frac{1}{4}, \frac{1}{2}\right),\left(1-k+\frac{\alpha}{4}, \frac{\alpha}{2}\right) \\
\left(-\frac{1}{2}, 1\right),\left(\frac{1}{4}, \frac{1}{2}\right)
\end{array}\right] \\
=\left(\frac{|x|}{\lambda} t^{-\frac{\alpha}{2}}\right)^{-\frac{1}{2}} \varphi\left(-\frac{\alpha}{2}, \frac{\alpha}{2}+1-k ;-\frac{|x|}{\lambda} t^{-\frac{\alpha}{2}}\right) .
\end{gathered}
$$


P r o o f. By the property of $H_{p . q}^{m, n}$-function [7, Property 2.2], with $m=n=p=2$ and $q=0$, and definition of the $H$-function (1.15), we have

$$
\begin{gathered}
H_{2,2}^{2,0}\left[\frac{|x|}{\lambda} t^{-\frac{\alpha}{2}} \mid \begin{array}{c}
\left(\frac{1}{4}, \frac{1}{2}\right),\left(1-k+\frac{\alpha}{4}, \frac{\alpha}{2}\right) \\
\left(-\frac{1}{2}, 1\right),\left(\frac{1}{4}, \frac{1}{2}\right)
\end{array}\right] \\
=H_{1,1}^{1,0}\left[\frac{|x|}{\lambda} t^{-\frac{\alpha}{2}} \mid \begin{array}{c}
\left(1-k+\frac{\alpha}{4}, \frac{\alpha}{2}\right) \\
\left(-\frac{1}{2}, 1\right)
\end{array}\right] \\
=\frac{1}{2 \pi i} \int_{L} \frac{\Gamma\left(-\frac{1}{2}+\tau\right)}{\Gamma\left(1-k+\frac{\alpha}{2}+\tau / 2\right)}\left(\frac{|x|}{\lambda} t^{-\frac{\alpha}{2}}\right)^{-\tau} d \tau,
\end{gathered}
$$

Using usual technique and evaluating residues of the integrand at poles $\tau_{j}=-j+\frac{1}{2}$ of the gamma function $\Gamma\left(-\frac{1}{2}+\tau\right)$ and taking (1.17) into account we deduce (4.1), which proves the lemma.

Setting $m=1$ and using Lemma 4 , from Theorems 3 and 4 we deduce the corresponding assertions for equation (1.6).

Theorem 5. If $0<\alpha \leq 1$ and $\lambda>0$, then the Cauchy-type problem

$$
\left(D_{0+, t}^{\alpha} u\right)(x, t)=\lambda^{2} \frac{\partial^{2} u(x, t)}{\partial x^{2}}, \quad\left(D_{0+, t}^{\alpha-1} u\right)(x, 0+)=f(x) \quad(x \in \mathbb{R}, t>0)
$$

is solvable, and its explicit solution is given by

$$
\begin{gathered}
u(x, t)=\int_{\mathbb{R}} G_{1}^{\alpha}(x-\tau, t) f(\tau) d \tau, \\
G_{1}^{\alpha}(x, t)=\frac{1}{2 \lambda} t^{\frac{\alpha}{2}-1} \varphi\left(-\frac{\alpha}{2}, \frac{\alpha}{2} ;-\frac{|x|}{\lambda} t^{-\frac{\alpha}{2}}\right),
\end{gathered}
$$

provided that the integral in the right-hand side of (4.4) is convergent.

Theorem 6. If $1<\alpha<2$ and $\lambda>0$, then the Cauchy-type problem for equation (1.6) with the initial conditions

$$
\left(D_{0+, t}^{\alpha-1} u\right)(x, 0+)=f_{1}(x), \quad\left(D_{0+, t}^{\alpha-2} u\right)(x, 0+)=f_{2}(x) \quad(x \in \mathbb{R})
$$

is solvable, and its explicit solution has the form

$$
u(x, t)=\int_{\mathbb{R}}\left[G_{1}^{\alpha}(x-\tau, t) f_{1}(\tau)+G_{2}^{\alpha}(x-\tau, t) f_{2}(\tau)\right] d \tau,
$$


provided that the integral in the right-hand side of (4.7) is convergent. Here $G_{1}^{\alpha}(x, t)$ is given by (4.5), and

$$
G_{2}^{\alpha}(x, t)=\frac{1}{2 \lambda} t^{\frac{\alpha}{2}-2} \varphi\left(-\frac{\alpha}{2}, \frac{\alpha}{2}-1 ;-\frac{|x|}{\lambda} t^{-\frac{\alpha}{2}}\right) .
$$

REMARK 3. The explicit solution (4.4) of the Cauchy-type problem (4.3) coincides with the known solution obtained in [12, Example 4.4].

\section{Behavior of solutions for large $x$}

We investigate a behavior of solutions (3.16) and (3.19), as $|x| \rightarrow \infty$. For this we find an asymptotic estimate of the $H_{2,2}^{2,0}$-functions in (3.17) and (3.20).

Lemma 5. Let $m \in \mathbb{N}, 0<\alpha<2, k=1$ for $0<\alpha \leq 1$, while $k=1,2$ for $1<\alpha<2$. Then for any fixed $t>0$ there hold the following asymptotic estimates:

$$
\begin{gathered}
H_{2,2}^{2,0}\left[\frac{|x|}{\lambda} t^{-\frac{\alpha}{2}} \mid \begin{array}{c}
\left(\frac{m}{4}, \frac{1}{2}\right),\left(1-k-\frac{\alpha(m-2)}{4}, \frac{\alpha}{2}\right) \\
\left(\frac{m}{2}-1,1\right),\left(\frac{1}{2}-\frac{m}{4}, \frac{1}{2}\right)
\end{array}\right] \\
=A_{k} \exp \left[\begin{array}{c}
(2-\alpha) \\
2
\end{array}\left(\frac{\alpha}{2}\right)^{\frac{\alpha}{2-\alpha}}\left(\frac{|x|}{\lambda t^{\frac{\alpha}{2}}}\right)^{\frac{2}{2-\alpha}}\right] \\
\times\left(\frac{|x|}{\lambda t^{\frac{\alpha}{2}}}\right)^{\frac{2}{2-\alpha}\left[\frac{\alpha(m-2)}{4}+k-1\right]}\left[1+O\left(\frac{t^{\frac{\alpha}{2}}}{|x|}\right)^{\frac{2}{2-\alpha}}\right] \quad(|x| \rightarrow \infty, k=1,2),
\end{gathered}
$$

where $A_{k}(k=1,2)$ are certain constants.

P r o o f. Formula (5.1) follows from the exponential estimate at infinity of more general $H_{p, q}^{q, 0}$-function given in [7, Theorem 1.10].

From (5.1) we deduce the asymptotic relations for functions $G_{1}^{\alpha}(x, t)$ and $G_{2}^{\alpha}(x, t)$ in (3.17) and (3.20): for any fixed $t>0$ we have, as $|x| \rightarrow \infty$

$$
\begin{gathered}
G_{k}^{\alpha}(x, t)=B_{k} \exp \left[-\frac{(2-\alpha)}{2}\left(\frac{\alpha}{2}\right)^{\frac{\alpha}{2-\alpha}}\left(\frac{|x|}{\lambda t^{\frac{\alpha}{2}}}\right)^{\frac{2}{2-\alpha}}\right] \\
\times|x|^{\frac{2-m}{2}} t^{-k-\frac{\alpha(m-2)}{4}}\left(\frac{|x|}{\lambda t^{\frac{\alpha}{2}}}\right)^{\frac{2}{2-\alpha}\left[\frac{\alpha(m-2)}{4}+k-1\right]}\left[1+O\left(\frac{t^{\frac{\alpha}{2}}}{|x|}\right)^{\frac{2}{2-\alpha}}\right],
\end{gathered}
$$


with

$$
B_{k}=\frac{2^{-m}}{\lambda^{1+\frac{m}{2}} \pi^{\frac{m-1}{2}}} A_{k} \quad(k=1,2),
$$

where $k=1$ for $0<\alpha \leq 1$, while $k=1,2$ for $1<\alpha<2$.

Since $0<\alpha<2$, then it follows from (5.2) that $G_{1}^{\alpha}(x, t)$ and $G_{2}^{\alpha}(x, t)$ for any fixed $t>0$ tend to zero, as $|x| \rightarrow \infty$,

$$
\lim _{|x| \rightarrow \infty} G_{1}^{\alpha}(x, t)=\lim _{|x| \rightarrow \infty} G_{2}^{\alpha}(x, t)=0 \quad\left(x \in \mathbb{R}^{m}\right) .
$$

Then from (3.16) and (3.19) we obtain the following result.

Theorem 7. Let $0<\alpha<2, m \in \mathbb{N}$ and $\lambda>0$.

(a) If $0<\alpha \leq 1$, then solution (3.16) of the Cauchy-type problem (3.15) for any fixed $t>0$ tends to zero, as $|x| \rightarrow \infty$ :

$$
\lim _{|x| \rightarrow \infty} u(x, t)=0 \quad\left(x \in \mathbb{R}^{m}\right) .
$$

(b) If $1<\alpha<2$, then solution (3.19) of the Cauchy-type problem (1.1), (3.18) for any fixed $t>0$ tends to zero, as $|x| \rightarrow \infty$ :

$$
\lim _{|x| \rightarrow \infty} u(x, t)=0 \quad\left(x \in \mathbb{R}^{m}\right) .
$$

Corollary 7. Let $0<\alpha<2$ and $\lambda>0$.

(a) If $0<\alpha \leq 1$, then solution (4.4) of the Cauchy-type problem (4.3) for any fixed $t>0$ tends to zero, as $|x| \rightarrow \infty$ :

$$
\lim _{|x| \rightarrow \infty} u(x, t)=0 \quad(x \in \mathbb{R}) .
$$

(b) If $1<\alpha<2$, then solution (4.7) of the Cauchy-type problem (1.6), (4.6) for any fixed $t>0$ tends to zero, as $|x| \rightarrow \infty$ :

$$
\lim _{|x| \rightarrow \infty} u(x, t)=0 \quad(x \in \mathbb{R}) .
$$

REMARK 4. Relations (5.5) and (5.6) show that solutions (3.16) and (3.19) of the Cauchy-type problems for the partial fractional differential equation (1.1) of order $0<\alpha \leq 1$ and $1<\alpha<2$, with the respective initial conditions (3.15) and (3.18), tend to zero, as $x$ tends to infinity. This result show that solutions of the above problems have the same property as solutions of the Cauchy problem for the heat equation (1.4) and for the wave equation (1.5). This property usually put as the initial condition of the Cauchy problem for the heat and wave equations (1.4) and (1.5); for example, see [19]. 


\section{Examples}

Here we give examples of solutions of the Cauchy-type problems (1.1), (1.7) and (1.6), (1.7) and illustrate the obtained solutions in the case $m=1$ and $f(x)=\frac{\sin x}{x}$ in graphs by using the program Mathematica. We begin with the case $\alpha=1 / 2$.

EXAmple 1. Consider the Cauchy-type problem (3.15) with $\alpha=1 / 2$ :

$$
\begin{gathered}
\left(D_{0+, t}^{1 / 2} u\right)(x, t)=\lambda^{2}\left(\Delta_{x} u\right)(x, t), \\
\left(D_{0+, t}^{-1 / 2} u\right)(x, 0+)=f(x) \quad\left(x \in \mathbb{R}^{m}, t>0\right)
\end{gathered}
$$

By Theorem 3, its solution has the form

$$
u(x, t)=\int_{\mathbb{R}^{m}} G_{1}^{1 / 2}(x-\tau, t) f(\tau) d \tau,
$$

where $G_{1}^{1 / 2}(x, t)$ is given by (3.17) with $\alpha=1 / 2$. When $m=1$, then by Theorem 5 the Cauchy-type problem

$$
\left(D_{0+, t}^{\alpha} u\right)(x, t)=\lambda^{2} \frac{\partial^{2} u(x, t)}{\partial x^{2}}, \quad\left(D_{0+, t}^{-1 / 2} u\right)(x, 0+)=f(x) \quad(x \in \mathbb{R}, t>0)
$$

has the solution

$$
u(x, t)=\int_{\mathbb{R}} G_{1}^{1 / 2}(x-\tau, t) f(\tau) d \tau,
$$

where $G_{1}^{1 / 2}(x, t)$ is given by (4.5) with $\alpha=1 / 2$.

If $f(x)$ is infinitely differentiable on $\mathbb{R}^{m}$, then by Corollary 5

$$
u(x, t)=t^{-1 / 2} \sum_{j=0}^{\infty} \frac{\left(\lambda^{2} t^{1 / 2}\right)^{j}}{\Gamma\left(\frac{j+1}{2}\right)}\left(\Delta_{x}^{j} f_{k}\right)(x)
$$

and

$$
u(x, t)=t^{-1 / 2} \sum_{j=0}^{\infty} \frac{\left(\lambda^{2} t^{1 / 2}\right)^{j}}{\Gamma\left(\frac{j+1}{2}\right)} f^{(2 j)}(x)
$$

are solutions of problem (6.1) and (6.3), respectively. 


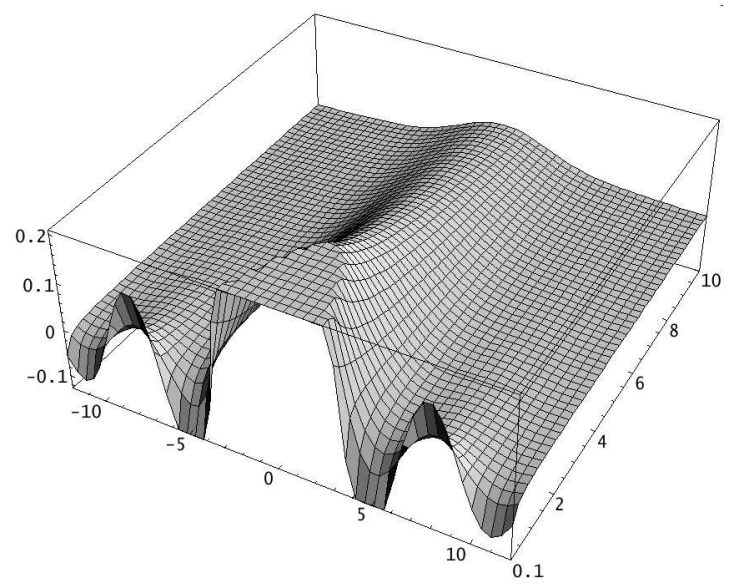

Figure 1:

Using the program Mathematica, we construct the graph of solution (6.6) with $\lambda=1$ and $f(x)=\frac{\sin x}{x}$ in Figure 1.

Example 2. Consider the Cauchy problem for the diffusion (heat) equation (1.4):

$$
\frac{\partial u(x, t)}{\partial t}=\lambda^{2}\left(\Delta_{x} u\right)(x, t), \quad u(x, 0)=f(x) \quad\left(x \in \mathbb{R}^{m}, t>0\right) .
$$

By Theorem 3, this problem has the solution

$$
u(x, t)=\int_{\mathbb{R}^{m}} G_{1}^{1}(x-\tau, t) f(\tau) d \tau .
$$

Lemma 6. $G_{1}^{\alpha}(x, t)$ in (3.17) for $\alpha=1$ has the form

$$
G_{1}^{1}(x, t)=\frac{1}{(2 \lambda \sqrt{\pi})^{m}} t^{-\frac{m}{2}} e^{-\frac{|x|^{2}}{4 \lambda^{2} t}} \quad\left(x \in \mathbb{R}^{m}, t>0\right) .
$$

P r o o f. By (3.17),

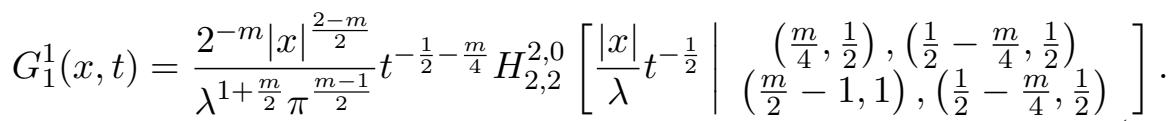


Using the properties of the $H$-function (1.15) [7, (2.1.2) and (2.1.5)] and applying the same arguments, as in the proof of (4.2), we have

$$
\begin{gathered}
H_{2,2}^{2,0}\left[\frac{|x|}{\lambda} t^{-\frac{1}{2}} \mid \begin{array}{c}
\left(\frac{m}{4}, \frac{1}{2}\right),\left(\frac{1}{2}-\frac{m}{4}, \frac{1}{2}\right) \\
\left(\frac{m}{2}-1,1\right),\left(\frac{1}{2}-\frac{m}{4}, \frac{1}{2}\right)
\end{array}\right] \\
=H_{1,1}^{1,0}\left[\frac{|x|}{\lambda} t^{-\frac{1}{2}} \mid \begin{array}{c}
\left(\frac{m}{4}, \frac{1}{2}\right) \\
\left(\frac{m}{2}-1,1\right)
\end{array}\right] \\
=\left(\frac{|x|}{\lambda} t^{-\frac{1}{2}}\right)^{\frac{m}{2}-1} H_{1,1}^{1,0}\left[\frac{|x|}{\lambda} t^{-\frac{1}{2}} \mid \begin{array}{c}
\left(\frac{1}{2}, \frac{1}{2}\right) \\
(0,1)
\end{array}\right] \\
=\left(\frac{|x|}{\lambda} t^{-\frac{1}{2}}\right)^{\frac{m}{2}-1} \varphi\left(-\frac{1}{2}, \frac{1}{2} ;-\frac{|x|}{\lambda} t^{-\frac{1}{2}}\right)
\end{gathered}
$$

By the definition (1.17) of the Wright function, it is directly verified that

$$
\varphi\left(-\frac{1}{2}, \frac{1}{2} ; z\right)=\frac{1}{\sqrt{\pi}} e^{-\frac{z^{2}}{4}}(z \in \mathbb{C}) .
$$

According to (6.12), relation (6.11) takes the form

$$
H_{2,2}^{2,0}\left[\frac{|x|}{\lambda} t^{-\frac{1}{2}} \mid \begin{array}{c}
\left(\frac{m}{4}, \frac{1}{2}\right),\left(\frac{1}{2}-\frac{m}{4}, \frac{1}{2}\right) \\
\left(\frac{m}{2}-1,1\right),\left(\frac{1}{2}-\frac{m}{4}, \frac{1}{2}\right)
\end{array}\right]=|x|^{\frac{m}{2}-1} \lambda^{1-\frac{m}{2}} \pi^{-\frac{1}{2}} t^{\frac{1}{2}-\frac{m}{4}} e^{-\frac{|x|^{2}}{4 \lambda t}} .
$$

Substitution of (6.13) into (6.10) yields (6.9), and lemma is proved.

By (6.9), relation (6.8) has the form

$$
u(x, t)=\int_{\mathbb{R}^{m}} G(x-\tau, t) f(\tau) d \tau, \quad G(x, t)=\frac{1}{(2 \lambda \sqrt{\pi})^{m}} t^{-\frac{m}{2}} e^{-\frac{|x|^{2}}{4 \lambda^{2} t}} .
$$

This solution of the Cauchy problem (6.7) is well known: for example, see [19, p.230 and p.482].

In particular, when $m=1$ the Cauchy problem

$$
\frac{\partial u(x, t)}{\partial t}=\lambda^{2} \frac{\partial^{2} u}{\partial x^{2}}(x, t), \quad u(x, 0)=f(x) \quad(x \in \mathbb{R}, t>0)
$$

has the solution

$$
u(x, t)=\int_{\mathbb{R}} G(x-\tau, t) f(\tau) d \tau, \quad G(x, t)=\frac{1}{2 \lambda \sqrt{\pi}} t^{-\frac{1}{2}} e^{-\frac{|x|^{2}}{4 \lambda^{2} t}} .
$$




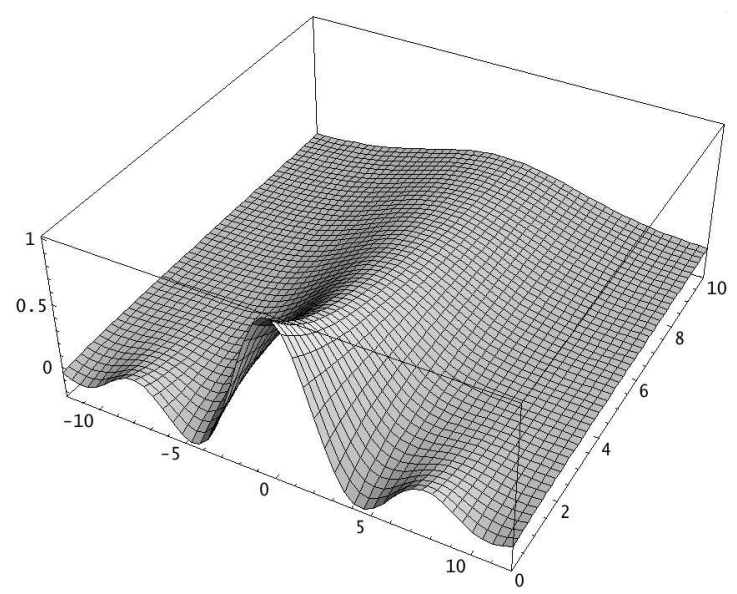

Figure 2:

Using the program Mathematica, we present the graph of solution (6.16) with $\lambda=1$ and $f(x)=\frac{\sin x}{x}$ in Figure 2 .

Example 3. Consider the Cauchy-type problem (1.1), (3.18) with $\alpha=$ $3 / 2$ :

$$
\begin{array}{r}
\left(D_{0+, t}^{3 / 2} u\right)(x, t)=\lambda^{2}\left(\Delta_{x} u\right)(x, t) \quad\left(x \in \mathbb{R}^{m}, t>0\right), \\
\left(D_{0+, t}^{1 / 2} u\right)(x, 0+)=f(x), \quad\left(D_{0+, t}^{-1 / 2} u\right)(x, 0+)=g(x) \quad\left(x \in \mathbb{R}^{m}\right) .
\end{array}
$$

By Theorem 3, its solution has the form

$$
u(x, t)=\int_{\mathbb{R}^{m}}\left[G_{1}^{3 / 2}(x-\tau, t) f(\tau)+G_{2}^{3 / 2}(x-\tau, t) g(\tau)\right] d \tau,
$$

where $G_{1}^{3 / 2}(x, t)$ and $G_{2}^{3 / 2}(x, t)$ are given respectively by (3.17) and (3.20) with $\alpha=3 / 2$. When $m=1$, then by Theorem 6 the Cauchy-type problem

$$
\begin{gathered}
\left(D_{0+, t}^{3 / 2} u\right)(x, t)=\lambda^{2} \frac{\partial^{2} u(x, t)}{\partial x^{2}} \quad(x \in \mathbb{R} ; t>0) \\
\left(D_{0+, t}^{1 / 2} u\right)(x, 0+)=f(x), \quad\left(D_{0+, t}^{-1 / 2} u\right)(x, 0+)=g(x)(x \in \mathbb{R} ; t>0)
\end{gathered}
$$
has the solution

$$
u(x, t)=\int_{\mathbb{R}}\left[G_{1}^{3 / 2}(x-\tau, t) f(\tau)+G_{2}^{3 / 2}(x-\tau, t) g(\tau)\right] d \tau,
$$


where $G_{1}^{3 / 2}(x, t)$ and $G_{2}^{3 / 2}(x, t)$ are given respectively by (4.5) and (4.8) with $\alpha=3 / 2$.

If $f(x)$ and $g(x)$ are infinitely differentiable on $\mathbb{R}^{m}$, then by Corollary 6

$$
u(x, t)=t^{1 / 2} \sum_{j=0}^{\infty} \frac{\left(\lambda^{2} t^{3 / 2}\right)^{j}}{\Gamma\left(\frac{3 j}{2}+\frac{3}{2}\right)}\left(\Delta_{x}^{j} f\right)(x)+t^{-1 / 2} \sum_{j=0}^{\infty} \frac{\left(\lambda^{2} t^{3 / 2}\right)^{j}}{\Gamma\left(\frac{3 j}{2}+\frac{1}{2}\right)}\left(\Delta_{x}^{j} g\right)(x)
$$

and

$$
u(x, t)=t^{1 / 2} \sum_{j=0}^{\infty} \frac{\left(\lambda^{2} t^{3 / 2}\right)^{j}}{\Gamma\left(\frac{3 j}{2}+\frac{3}{2}\right)} f^{(2 j)}(x)+t^{-1 / 2} \sum_{j=0}^{\infty} \frac{\left(\lambda^{2} t^{3 / 2}\right)^{j}}{\Gamma\left(\frac{3 j}{2}+\frac{1}{2}\right)} g^{(2 j)}(x)
$$

are solutions of problem (6.17), (6.18) and (6.20), (6.21), respectively.

Using the program Mathematica, we give the graph of solution (6.24) with $\lambda=1, f(x)=0$ and $g(x)=\frac{\sin x}{x}$ in Figure 3 .

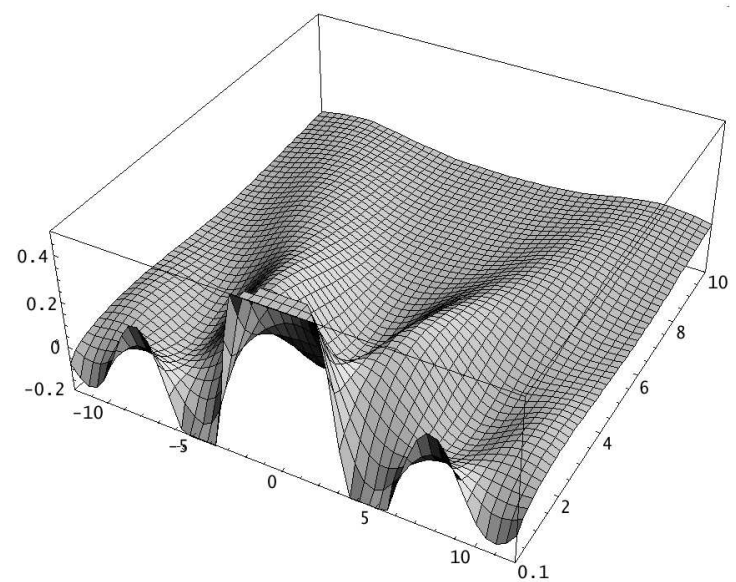

Figure 3:

ExAmPLE 4. Consider the Cauchy problem for the wave equation (1.5):

$$
\begin{gathered}
\frac{\partial^{2} u(x, t)}{\partial t^{2}}=\lambda^{2}\left(\Delta_{x} u\right)(x, t), \\
u(x, 0)=g(x), \frac{\partial u(x, 0)}{\partial t}=f(x) \quad\left(x \in \mathbb{R}^{m}, t>0\right) .
\end{gathered}
$$


By Corollary 1, this problem has the solution

$$
\begin{gathered}
u(x, t)=\frac{t^{\alpha-1}}{(2 \pi)^{m}} \int_{\mathbb{R}^{m}} E_{\alpha, \alpha}\left(-\lambda^{2}|\sigma|^{2} t^{\alpha}\right)\left(\mathcal{F}_{x} f\right)(\sigma) e^{-i x \cdot \sigma} d \sigma+ \\
+\frac{t^{\alpha-2}}{(2 \pi)^{m}} \int_{\mathbb{R}^{m}} E_{\alpha, \alpha-1}\left(-\lambda^{2}|\sigma|^{2} t^{\alpha}\right)\left(\mathcal{F}_{x} g\right)(\sigma) e^{-i x \cdot \sigma} d \sigma .
\end{gathered}
$$

When $m=1$, the Cauchy problem

$$
\begin{gathered}
\frac{\partial^{2} u(x, t)}{\partial t^{2}}=\lambda^{2} \frac{\partial^{2} u(x, t)}{\partial x^{2}} \\
u(x, 0)=g(x), \frac{\partial u(x, 0)}{\partial t}=f(x) \quad(x \in \mathbb{R}, t>0)
\end{gathered}
$$

has the solution

$$
\begin{gathered}
u(x, t)=\frac{t^{\alpha-1}}{2 \pi} \int_{\mathbb{R}} E_{\alpha, \alpha}\left(-\lambda^{2}|\sigma|^{2} t^{\alpha}\right)\left(\mathcal{F}_{x} f\right)(\sigma) e^{-i x \sigma} d \sigma+ \\
+\frac{t^{\alpha-2}}{2 \pi} \int_{\mathbb{R}} E_{\alpha, \alpha-1}\left(-\lambda^{2}|\sigma|^{2} t^{\alpha}\right)\left(\mathcal{F}_{x} g\right)(\sigma) e^{-i x \sigma} d \sigma .
\end{gathered}
$$

If $f(x)$ and $g(x)$ are infinitely differentiable on $\mathbb{R}^{m}$, then by Corollary 4 ,

$$
u(x, t)=t \sum_{j=0}^{\infty} \frac{(\lambda t)^{2 j}}{(2 j+1) !}\left(\Delta_{x}^{j} f\right)(x)+\sum_{j=0}^{\infty} \frac{(\lambda t)^{2 j}}{(2 j) !}\left(\Delta_{x}^{j} g\right)(x)
$$

and

$$
u(x, t)=t \sum_{j=0}^{\infty} \frac{(\lambda t)^{2 j}}{(2 j+1) !} f^{(2 j)}(x)+\sum_{j=0}^{\infty} \frac{(\lambda t)^{2 j}}{(2 j) !} g^{(2 j)}(x)
$$

are solutions of Cauchy problems (6.25) and (6.27), respectively.

Denoting by $F(x)$ a primitive of $f(x)$ and using the Tailor expansions of $f(x)$ and $g(x)$ in a neighborhood of $x \in \mathbb{R}$, we have for solution (6.30)

$$
\begin{aligned}
u(x, t) & =\frac{1}{\lambda} \sum_{j=0}^{\infty} \frac{(\lambda t)^{2 j+1}}{(2 j+1) !} F^{(2 j+1)}(x)+\sum_{j=0}^{\infty} \frac{(\lambda t)^{2 j}}{(2 j) !} g^{(2 j)}(x) \\
= & \frac{1}{2 \lambda}\left(\sum_{i=0}^{\infty} \frac{(\lambda t)^{i}}{i !} F^{(i)}(x)-\sum_{i=0}^{\infty} \frac{(-\lambda t)^{i}}{i !} F^{(i)}(x)\right)
\end{aligned}
$$




$$
\begin{gathered}
+\frac{1}{2}\left(\sum_{i=0}^{\infty} \frac{(\lambda t)^{i}}{i !} g^{(i)}(x)+\sum_{i=0}^{\infty} \frac{(-\lambda t)^{i}}{i !} g^{(i)}(x)\right) \\
=\frac{F(x+\lambda t)-F(x-\lambda t)}{2 \lambda}+\frac{f(x+\lambda t)+f(x-\lambda t)}{2} .
\end{gathered}
$$

Hence (6.30) takes the form

$$
u(x . t)=\frac{1}{2 \lambda} \int_{x-\lambda t}^{x+\lambda t} f(\tau) d \tau+\frac{g(x+\lambda t)+g(x-\lambda t)}{2},
$$

which coincides with the well known solution of the Cauchy problem (6.27): for example, see [19, p. 56].

Using the program Mathematica, we give the graph of solution (6.31) with $\lambda=1, f(x)=0$ and $g(x)=\frac{\sin x}{x}$ in Figure 4.

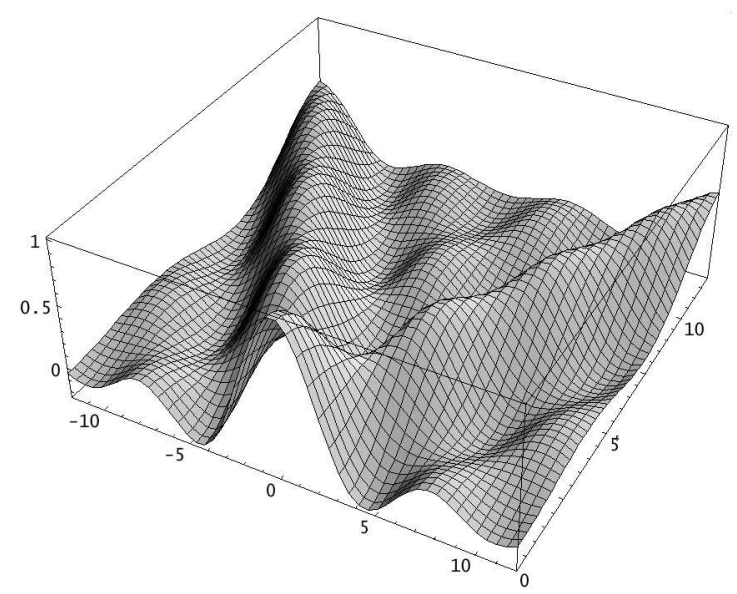

Figure 4:

EXAmple 5. Consider the Cauchy-type problem (1.1), (1.7) with $\alpha=$ $5 / 2$ :

$$
\begin{gathered}
\left(D_{0+, t}^{5 / 2} u\right)(x, t)=\lambda^{2}\left(\Delta_{x} u\right)(x, t) \quad\left(x \in \mathbb{R}^{m}, t>0\right), \\
\left(D_{0+, t}^{3 / 2} u\right)(x, 0+)=f(x), \quad\left(D_{0+, t}^{1 / 2} u\right)(x, 0+)=g(x), \\
\left(D_{0+, t}^{-1 / 2} u\right)(x, 0+)=h(x) .
\end{gathered}
$$


By Theorem 1, its solution has the form

$$
\begin{gathered}
u(x, t)=\frac{t^{3 / 2}}{(2 \pi)^{m}} \int_{\mathbb{R}^{m}} E_{5 / 2,5 / 2}\left(-\lambda^{2}|\sigma|^{2} t^{5 / 2}\right)\left(\mathcal{F}_{x} f\right)(\sigma) e^{-i x \cdot \sigma} d \sigma+ \\
+\frac{t^{1 / 2}}{(2 \pi)^{m}} \int_{\mathbb{R}^{m}} E_{5 / 2,3 / 2}\left(-\lambda^{2}|\sigma|^{2} t^{5 / 2}\right)\left(\mathcal{F}_{x} g\right)(\sigma) e^{-i x \cdot \sigma} d \sigma+ \\
+\frac{t^{-1 / 2}}{(2 \pi)^{m}} \int_{\mathbb{R}^{m}} E_{5 / 2,1 / 2}\left(-\lambda^{2}|\sigma|^{2} t^{5 / 2}\right)\left(\mathcal{F}_{x} h\right)(\sigma) e^{-i x \cdot \sigma} d \sigma .
\end{gathered}
$$

Relation (6.34) with $m=1$ yields the explicit solution of the Cauchy-type problem

$$
\begin{gathered}
\left(D_{0+, t}^{5 / 2} u\right)(x, t)=\lambda^{2} \frac{\partial^{2} u(x, t)}{\partial x^{2}} \quad(x \in \mathbb{R}, t>0), \\
\left(D_{0+, t}^{3 / 2} u\right)(x, 0+)=f(x), \quad\left(D_{0+, t}^{1 / 2} u\right)(x, 0+)=g(x), \\
\left(D_{0+, t}^{-1 / 2} u\right)(x, 0+)=h(x) .
\end{gathered}
$$

If $f(x), g(x)$ and $h(x)$ are infinitely differentiable on $\mathbb{R}^{m}$, then by Theorem 2 , solution of $(6.32),(6.33)$ is given by

$$
\begin{gathered}
u(x, t)=t^{3 / 2} \sum_{j=0}^{\infty} \frac{\left(\lambda^{2} t^{5 / 2}\right)^{j}}{\Gamma\left(\frac{5 j}{2}+\frac{5}{2}\right)}\left(\Delta_{x}^{j} f\right)(x) \\
+t^{1 / 2} \sum_{j=0}^{\infty} \frac{\left(\lambda^{2} t^{5 / 2}\right)^{j}}{\Gamma\left(\frac{5 j}{2}+\frac{3}{2}\right)}\left(\Delta_{x}^{j} g\right)(x)+t^{-1 / 2} \sum_{j=0}^{\infty} \frac{\left(\lambda^{2} t^{5 / 2}\right)^{j}}{\Gamma\left(\frac{5 j}{2}+\frac{1}{2}\right)}\left(\Delta_{x}^{j} h\right)(x) .
\end{gathered}
$$

In particular, when $m=1$, the solution of problem (6.35), (6.36) takes the form

$$
\begin{gathered}
u(x, t)=t^{3 / 2} \sum_{j=0}^{\infty} \frac{\left(\lambda^{2} t^{5 / 2}\right)^{j}}{\Gamma\left(\frac{5 j}{2}+\frac{5}{2}\right)} f^{(2 j)}(x)+ \\
+t^{1 / 2} \sum_{j=0}^{\infty} \frac{\left(\lambda^{2} t^{5 / 2}\right)^{j}}{\Gamma\left(\frac{5 j}{2}+\frac{3}{2}\right)} g^{(2 j)}(x)+t^{-1 / 2} \sum_{j=0}^{\infty} \frac{\left(\lambda^{2} t^{5 / 2}\right)^{j}}{\Gamma\left(\frac{5 j}{2}+\frac{1}{2}\right)} h^{(2 j)}(x) .
\end{gathered}
$$

Using the program Mathematica, we construct the graph of solution (6.38) with $\lambda=1, f(x)=g(x)=0$ and $h(x)=\frac{\sin x}{x}$ in Figure 5. 
Acknowledgements. This work was supported, in part, by Belarusian Fundamental Research Fund (project F05MC-050), and by DGUI of G.A.CC and by ULL Research Funds.

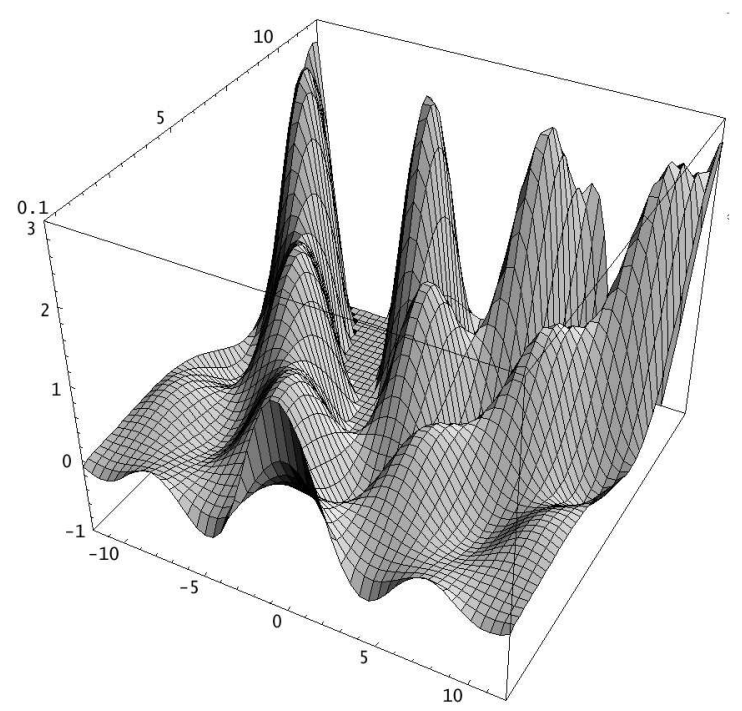

Figure 5:

\section{References}

[1] A. C a r p i n t r i, F. M a i n a r d i (Eds), Fractal and Fractional Calculus in Continuum Mechanics, CIMS Course and Lectures 378, Springer-Verlag, Berlin (1997).

[2] V.A. D i t k i n, A. P. P r u d $\mathrm{n} \mathrm{i} \mathrm{k} \mathrm{o} \mathrm{v}$, Integral Transforms and Operational Calculus, Pergamon Press, Oxford (1965).

[3] A. E r d e l y i, W. M a g n u s, F. O b e r h e t t i n g e r, F.G. T r i c o m i, Higher Transcendental Functions, Vol. II, McGraw-Hill, New York - Toronto - London (1953); Reprinted: Krieger, Melbourne, Florida (1981).

[4] A. E r d e l y i, W. M a g n u s, F. O b e r h e t t i n g e r, F.G. T r i c o m i, Higher Transcendental Functions, Vol. III, McGraw-Hill, New York - Toronto - London (1954); Reprinted: Krieger, Melbourne, Florida (1981).

[5] R. H i l f e r (Ed.), Applications of Fractional Calculus, WSPC, Singapore (2000). 
[6] R. H i $1 \mathrm{f}$ e r, Fractional diffusion based on Riemann-Liouville fractional derivatives, J. Phys. Chem. B 104, No. 3 (2000), 914-924.

[7] A. A. K i 1 b a s, M. S a i g o, H-Transforms. Theory and Applications, Chapman and Hall/CRC, Boca Raton, Florida (2004).

[8] A. A. K i l b a s, M. S a i g o, J. J. T r u j i 11 o, On the generalized Wright function. Dedicated to the 60th anniversary of Prof. Francesco Mainardi, Frac. Calc. Appl. Anal. 5, No. 4 (2002), 437-460.

[9] A. A. K i l b a s, J. J. T r u j i 11 o, Differential equations of fractional order: methods, results and problems, II, Applicable Analysis 81, No. 2 (2002), 435-493.

[10] A. M. M a t h a i, R. K. S a x e n a, The H-Function with Applications in Statistics and Other Disciplines), Halsted Press [John Wiley and Sons], New York-London-Sydney, 1978.

[11] S. M. N i k o l' s k i i, Course of Mathematical Analysis, (Russian), Vol. 2, Nauka, Moscow (1983).

[12] I. P o d l u b n y, Fractional Differential Equations, Mathematics in Sciences and Engineering, 198, Academic Press, San Diego (1999).

[13] A.P. P r u d n i k o v, Yu.A. B r y c h k o v, O.I. M a r i c h e v, Integrals and Series, Vol.2, Special Functions, Gordon and Breach Sci. Publ., New York (1986).

[14] A.P. P r u d $\mathrm{n}$ i k o v, Yu.A. B r y c h k o v, O.I. M a r i c h e $\mathrm{v}$, Integrals and Series, Vol.3, More Special Functions, Gordon and Breach Sci. Publ., New York (1990).

[15] S.G. S a m k o, A.A. K i 1 b a s, O.I. M a r i c h e v, Fractional Integrals and Derivatives. Theory and Applications, Gordon and Breach, New York at al. (1993).

[16] I. N. S n e d d o n, Fourier Transforms, McGraw-Hill, New York Toronto - London (1951); Reprinted in Dover Publication, New York (1995).

[17] H. M. S r i v a st a va a, K. C. G u p t a, S. L. G o y a l, The H-function of One and Two Variables with Applications, South Asian Publishers, New-Delhi-Madras (1982).

[18] E. M. S t e i n, G. W e i s s, Introduction to Fourier Analysis on Euclidean Spaces, Princeton Mathematical Series 32, Princeton Univ. Press, Princeton, New Jersey (1971).

[19] A. N. T i k h o n o v, A. A. S a m a r s k i i, Equations of Mathematical Physics (Russian), Nauka, Moscow (2004). 
${ }^{1}$ Department of Mathematics and Mechanics Belarusian State University 220050 Minsk, BELARUS

e-mail: kilbas@bsu.by

${ }^{2}$ Department of Matematical Analysis University of La Laguna 38271 La Laguna - Tenerife, SPAIN

e-mail: jtrujill@ull.es 